\title{
Perioperative outcomes for pediatric neurosurgical procedures: analysis of the National Surgical Quality Improvement Program-Pediatrics
}

\author{
Benjamin J. Kuo, BS,1-3 Joao Ricardo N. Vissoci, PhD, ${ }^{1,2}$ Joseph R. Egger, PhD, ${ }^{2}$ \\ Emily R. Smith, PhD, ${ }^{1,2}$ Gerald A. Grant, MD, ${ }^{6}$ Michael M. Haglund, MD, PhD, ${ }^{1,2,4}$ and \\ Henry E. Rice, MD2,5
}

1Division of Global Neurosurgery and Neuroscience and ${ }^{2}$ Global Health Institute, Duke University, Durham, North Carolina; ${ }^{3}$ Duke-NUS Medical School, Singapore; Departments of ${ }^{4}$ Neurosurgery and ${ }^{5}$ Surgery, Duke University Medical Center, Durham, North Carolina; and ${ }^{6}$ Department of Neurosurgery, Stanford University, Stanford, California

OBJECTIVE Existing studies have shown a high overall rate of adverse events (AEs) following pediatric neurosurgical procedures. However, little is known regarding the morbidity of specific procedures or the association with risk factors to help guide quality improvement (QI) initiatives. The goal of this study was to describe the 30 -day mortality and $A E$ rates for pediatric neurosurgical procedures by using the American College of Surgeons (ACS) National Surgical Quality Improvement Program-Pediatrics (NSQIP-Peds) database platform.

METHODS Data on 9996 pediatric neurosurgical patients were acquired from the 2012-2014 NSQIP-Peds participant user file. Neurosurgical cases were analyzed by the NSQIP-Peds targeted procedure categories, including craniotomy/ craniectomy, defect repair, laminectomy, shunts, and implants. The primary outcome measure was 30-day mortality, with secondary outcomes including individual AEs, composite morbidity (all AEs excluding mortality and unplanned reoperation), surgical-site infection, and unplanned reoperation. Univariate analysis was performed between individual AEs and patient characteristics using Fischer's exact test. Associations between individual AEs and continuous variables (duration from admission to operation, work relative value unit, and operation time) were examined using the Student t-test. Patient characteristics and continuous variables associated with any AE by univariate analysis were used to develop category-specific multivariable models through backward stepwise logistic regression.

RESULTS The authors analyzed 3383 craniotomy/craniectomy, 242 defect repair, 1811 laminectomy, and 4560 shunt and implant cases and found a composite overall morbidity of $30.2 \%, 38.8 \%, 10.2 \%$, and $10.7 \%$, respectively. Unplanned reoperation rates were highest for defect repair (29.8\%). The mortality rate ranged from $0.1 \%$ to $1.2 \%$. Preoperative ventilator dependence was a significant predictor of any AE for all procedure groups, whereas admission from outside hospital transfer was a significant predictor of any AE for all procedure groups except craniotomy/craniectomy.

CONCLUSIONS This analysis of NSQIP-Peds, a large risk-adjusted national data set, confirms low perioperative mortality but high morbidity for pediatric neurosurgical procedures. These data provide a baseline understanding of current expected clinical outcomes for pediatric neurosurgical procedures, identify the need for collecting neurosurgery-specific risk factors and complications, and should support targeted QI programs and clinical management interventions to improve care of children.

https://thejns.org/doi/abs/10.3171/2016.10.PEDS16414

KEY WORDS risk-adjustment; quality improvement; surgical outcome; National Surgical Quality Improvement Program

ABBREVIATIONS ACS = American College of Surgeons; $\mathrm{AE}=$ adverse event; $\mathrm{AOR}=$ adjusted $\mathrm{OR} ; \mathrm{ASA}=\mathrm{American}$ Society of Anesthesiologists; $\mathrm{Cl}=$ confidence interval; $\mathrm{CPT}=$ Current Procedural Terminology; IQR = interquartile range; NICU = neonatal intensive care unit; NSQIP = National Surgical Quality Improvement Program; NSQIPPeds = NSQIP-Pediatrics; OR = odds ratio; PICU = pediatric intensive care unit; $\mathrm{QI}=$ quality improvement; $\mathrm{SSI}$ = surgical site infection; WBC = white blood cell; $w R V U=$ work relative value unit.

SUBMITTED July 15, 2016. ACCEPTED October 7, 2016.

INCLUDE WHEN CITING Published online January 6, 2017; DOI: 10.3171/2016.10.PEDS16414. 
$\mathrm{P}$ EDIATRIC neurosurgery has one of the highest morbidity rates among pediatric surgical subspecialties. ${ }^{15}$ As health care costs rise, focus has increased on improving surgical outcomes through quality improvement (QI) initiatives. Advancements in the use of large risk-adjusted national databases allow for better delineation of relationships between patient risk factors and quality measures, such as postoperative adverse events (AEs). Understanding these correlations can support targeted QI programs as well as enhance clinical management.

The American College of Surgeons (ACS) National Surgical Quality Improvement Program (NSQIP) has created landmark transformations in the ability to reliably predict risk-adjusted postoperative complications for adults., Based on its success in the Veterans Affairs system and the private sector ${ }^{7}$ to provide surgical quality benchmarks and inform QI interventions, the ACS has partnered with the American Pediatric Surgical Association to create a similar database platform for the pediatric population, the National Surgical Quality Improvement Program-Pediatrics (NSQIP-Peds). ${ }^{3}$ Although NSQIP-Peds has numerous challenges unique to pediatric surgical outcomes, such as low mortality rates, differential clinical weighting of AEs, and the low volume of high-risk procedures, it provides an understanding of expected perioperative outcomes in children. ${ }^{12}$

To date, analysis of pediatric surgical subspecialty outcomes has been limited. ${ }^{16}$ Given the high morbidity following pediatric neurosurgical procedures, ${ }^{2}$ a critical analysis in this field at the procedural class level may identify contributors to AEs and identify opportunities for neurosurgical QI initiatives. Sherrod et al. recently reported various postoperative complications to be significant risk factors for unplanned readmission in pediatric neurosurgery using NSQIP-Peds, and identified actionable QI opportunities to mitigate risks of unplanned readmission. ${ }^{17}$ However, understanding of risk factors that are either associated with or causative of postoperative complications or unplanned reoperation remains quite limited. The purpose of this study was to describe outcomes following pediatric neurosurgical procedures using NSQIP-Peds. Furthermore, we wanted to identify patient risk factors and differences in measures of efficiency of care and procedural complexity associated with AEs.

\section{Methods \\ Data Source and Measures}

The data source for our study was the 2012-2014 participant use files of the NSQIP-Peds program. Currently, more than 100 hospitals are enrolled in the ACS NSQIPPeds program. At each participating institution, a systematic sampling process is used to ensure proportional case mix selection through 8-day sampling cycles. Readers are referred to previous reports of the NSQIP for case sampling procedures; ${ }^{2,12}$ however, in brief, 1400 pediatric surgical cases are collected at each participating institution annually based on 408 -day cycles, during each of which 35 cases are reviewed. The first 10 "essential" cases are collected in consecutive order utilizing the ACS NSQIPPeds Current Procedural Terminology (CPT) Code inclu- sion list. The subsequent 25 cases are procedure targeted and assigned to specific services by CPT (American Medical Association) code of the principal operative procedure. Continuing by date/time, each targeted case from the subspecialty CPT code inclusion list is selected until the following service limit is met for each subspecialty: general surgery 10 cases, neurosurgery 3 cases, orthopedic surgery 5 cases, otolaryngology 2 cases, plastic surgery 2 cases, and urological surgery 3 cases. To ensure that 35 cases per reporting cycle are collected each sampling cycle, additional cases in any subspecialty are collected consecutively to fill any service quota that was not met. At each site, a certified Surgical Clinical Reviewer, who received NSQIP-Peds training and quality control supervision to ensure reliable and accurate data capture, collects and enters patient data through medical chart review. ${ }^{18}$ More than 150 variables are collected, which include demographic information, preoperative risk factors, intraoperative variables, and 30-day postoperative mortality and morbidity events ("occurrences") for patients undergoing select surgical procedures. ${ }^{3}$ The data definitions and methodology for NSQIP-Peds have been described previously, and all occurrences are generally treated as dichotomous variables with unique data fields.,12,13 Data from each institution are submitted electronically to the ACS for processing and compiled into annual participant use files, which are accessible to all participating institutions.

\section{Case Selection}

As a study using only de-identified data, this project was granted exemption from review by the Duke University Institutional Review Board. Inclusion criteria for this study were any child (under 19 years of age) undergoing any neurosurgical procedures accrued within the data set. ${ }^{12}$ Any child with incomplete age data was excluded from the study. Pediatric neurosurgical cases were defined using CPT codes that are within the ACS NSQIP-Peds data set. Patients were delineated into the NSQIP-Peds designated neurosurgical procedure categories as used in January 2015: craniotomy/craniectomy, defect repair, laminectomy, and shunts and implants. Only CPT codes with captured patient cases were presented and analyzed. For example, in the defect repair procedure category, 7 CPT codes are included in this procedure targeted list, but only CPT codes 63704 and 63706, both for myelomeningocele repair, had recorded cases, whereas the others (CPT $61680,61682,61684,61686$, and 62201) had no reported cases. CPT codes for craniotomy/craniectomy (percentage of case volume in that category, in decreasing order) include: $61343(33.8 \%), 61510$ (21.5\%), 61518 (16.8\%), 61559 (14.6\%), 61550 (6.2\%), 61557 (3.8\%), 61558 (2.1\%), and $61545(1.2 \%)$. CPT codes for defect repairs include: $63706(51.7 \%)$ and $63704(48.3 \%)$. CPT codes for laminectomy include: 63200 (87.1\%), $63272(6.2 \%), 63287$ (2.2\%), $63286(2.0 \%), 63285$ (1.9\%), and $63273(0.6 \%)$. CPT codes for shunts and implants include: $62230(34.0 \%), 62223$ (23.8\%), 62225 (19.1\%), 62258 (9.1\%), 61210 (7.2\%), and $62362(6.8 \%)$. Note that no cases were extracted for the epilepsy category, because the CPT codes $(61885,61888$, $64568,64569)$ for this procedure group were only recently added to the NSQIP-Peds CPT code inclusion list. 


\section{Outcome Measures}

Our primary outcome measure was 30-day mortality. Secondary outcomes were individual 30-day AEs as well as composite morbidity as previously defined ${ }^{15}$ : superficial, deep, and organ space surgical site infections (SSI), superficial and deep wound dehiscence, systemic inflammatory response syndrome/sepsis/septic shock, unplanned reintubation, pneumonia, ventilator dependence $>24$ hours, pulmonary embolism, cardiac arrest, urinary tract infection, progressive renal insufficiency, acute renal failure, cerebrovascular accident, coma for $>24$ hours, seizure, nerve injury, venous thromboembolism, and intra- or postoperative blood transfusion.

SSIs were analyzed as a separate individual outcome. In addition, unplanned reoperation, which traditionally has not been included in measures of composite morbidity, was evaluated as an individual outcome, given its increasing use as a proxy for surgical quality in neurosurgery. ${ }^{9,10,19}$ Unplanned reoperation as defined by the NSQIP-Peds protocol excludes cases with preoperative or intraoperative documentation that specifies the need for a patient to return to the operating room following the principal operative procedure, such as return to the operating room for planned CSF diversion following repair of a spinal defect. Finally, we summarized patients who experienced any of the previously mentioned AEs, including unplanned reoperation. Each patient was only counted once, regardless of the total number of AEs experienced.

\section{System Efficiency and Procedural Complexity Measures}

Measures of efficiency of care included length of stay and time to operation since admission. These variables, along with other process measures, are increasingly used as proxies for quality of care and for their contribution to patient outcome. ${ }^{5}$ Measures of procedural complexity included work relative value unit (wRVU) and total operation time.

\section{Laboratory Data}

Collected laboratory data included baseline albumin, alkaline phosphatase, bilirubin, blood urea nitrogen, creatinine, hematocrit, international normalized ratio, platelets, partial thromboplastin time, prothrombin time, serum glutamic oxaloacetic transaminase, sodium, and white blood cells (WBCs).

\section{Statistical Analysis}

Statistical analyses were performed using Stata version 14.0 (Stata Corp). Associations of patient risk factors with the occurrence of any AE were analyzed with cross-tabulation and the Fisher's exact test. Univariate associations significant at the $\mathrm{p}<0.05$ level were used to construct multivariable models. Continuous variables (admission to operation days, length of hospital stay, operation time, and wRVU) are represented by their median with observed interquartile range (IQR), or by their means \pm standard deviation as appropriate. Associations between continuous variables and any AE were first analyzed using the Student t-test, with variables having a $p$ value $<0.05$ entered into each category-specific multivariable model with the exception of length of stay, which was only presented as a descriptive statistic. Adjusted odds ratios (ORs), 95\% confidence intervals (CIs), and $\mathrm{p}$ values were generated using backward stepwise multivariable logistic regression analysis at the $\mathrm{p}<0.05$ level.

Because of a substantial amount of missing laboratory data, we did not include any laboratory variable for multivariable analysis. Only hematocrit, platelets, and WBC variables had fewer than 33\% missing data, and we compared these variables between patients with no AEs versus patients with any AE by using the Student t-test.

For the craniotomy/craniectomy category, 11 patients were excluded out of 3382 cases for multivariable analysis due to missing data for American Society of Anesthesiologists (ASA) Class 4 or 5 (10 data points missing) and operation time (1 data point missing). In the defect repair category, 1 case was excluded out of 242 cases in the multivariable model due to missing data for ASA Class 4 or 5 . In the laminectomy category, no data were missing, and all 1811 cases were included in the multivariable model. In the shunts and implants category, no data were missing, and all 4560 cases were included in the multivariable model.

\section{Results \\ Demographics}

Data for a total of 9996 children who underwent an NSQIP-Peds targeted neurosurgical procedure between 2012 and 2014 were included in the analysis (Table 1). Among children who underwent a craniotomy/craniectomy procedure $(\mathrm{n}=3383)$, the median patient age was 5.8 years (IQR 1.3-12.4 years), 55.2\% were male, $76.5 \%$ were Caucasian, $9.7 \%$ were preterm, and $2.8 \%$ had ASA Class 4 or 5. The predominant admission source was from home with $85.5 \%$ of patients, and $8.2 \%$ were urgent or emergency cases. Among children who underwent a defect repair procedure, the median patient age was 1 day (IQR 1-1 day), $54.5 \%$ were male, $74.0 \%$ were Caucasian, $18.2 \%$ were preterm, and $9.9 \%$ were ASA Class 4 or 5 . More than half were outside hospital transfers (newborn intensive care unit [NICU], pediatric intensive care unit [PICU], inpatient), and urgent and emergency cases accounted for nearly $60 \%$ of all cases. Among children who underwent a laminectomy procedure, the median patient age was 5.5 years (IQR 1.4-10.3 years), $46.5 \%$ were male, $78.4 \%$ were Caucasian, $16.9 \%$ were preterm, and $0.7 \%$ had ASA Class 4 or 5. The predominant admission source was from home (97.6\%), and urgent and emergency cases accounted for $1.5 \%$. Among children who underwent shunt and implant procedures, the median age was 6.8 years (IQR 0.9-12.3 years), $56.8 \%$ were male, and $69.8 \%$ were Caucasian, with African American children representing a larger proportion than in other procedure categories with $18.7 \%$. Only $5.3 \%$ were preterm, and ASA Class 4 or 5 was noted in $6 \%$ of patients. A significant source of admission in this procedure category came from emergency rooms (40.1\%) and home (48.2\%). Urgent and emergency cases also represented a higher proportion than in other categories (46.7\%).

\section{Patient Risk Factors}

For all categories, more than $85 \%$ of patients had no 
TABLE 1. Demographic information by NSQIP-Peds neurosurgical procedure categories

\begin{tabular}{|c|c|c|c|c|}
\hline Demographics & Craniotomy/Craniectomy (\%) & Defect Repairs (\%) & Laminectomy (\%) & Shunts \& Implants (\%) \\
\hline Total cases & $3383(100)$ & $242(100)$ & $1811(100)$ & $4560(100)$ \\
\hline Male sex & $1867(55.2)$ & $132(54.5)$ & $842(46.5)$ & $2588(56.8)$ \\
\hline Hispanic ethnicity & $388(11.5)$ & $31(12.8)$ & $217(12)$ & $462(10.1)$ \\
\hline \multicolumn{5}{|l|}{ Race } \\
\hline Caucasian & $2589(76.5)$ & $179(74.0)$ & $1420(78.4)$ & $3184(69.8)$ \\
\hline African American & $319(9.4)$ & $26(10.7)$ & $128(7.1)$ & $852(18.7)$ \\
\hline Asian & $65(1.9)$ & $1(0.4)$ & $81(4.5)$ & $69(1.5)$ \\
\hline Pacific Islander & $7(0.2)$ & $1(0.4)$ & $4(0.2)$ & $11(0.2)$ \\
\hline Native American & $23(0.7)$ & $1(0.4)$ & $9(0.5)$ & $27(0.6)$ \\
\hline Other & $380(11.2)$ & $34(14.1)$ & $169(9.3)$ & $417(9.1)$ \\
\hline \multicolumn{5}{|l|}{ Age } \\
\hline$<29$ days & $11(0.3)$ & $220(90.9)$ & $19(1)$ & $280(6.1)$ \\
\hline 29-364 days & $936(27.7)$ & $12(5)$ & $346(19.1)$ & $939(20.6)$ \\
\hline $1-5$ yrs & $936(27.7)$ & $5(2.1)$ & $598(33)$ & $1117(24.5)$ \\
\hline $6-12$ yrs & $930(27.5)$ & $4(1.7)$ & $612(33.8)$ & $1242(27.2)$ \\
\hline $13-18$ yrs & $738(21.8)$ & $1(0.4)$ & $236(13)$ & $982(21.5)$ \\
\hline Preterm birth* & $327(9.7)$ & 44 (18.2) & $306(16.9)$ & $241(5.3)$ \\
\hline \multicolumn{5}{|l|}{ ASA class } \\
\hline 1 & $240(7.1)$ & $7(2.9)$ & $164(9.1)$ & $57(1.3)$ \\
\hline 2 & $1487(44)$ & $70(28.9)$ & $1042(57.5)$ & $1304(28.6)$ \\
\hline 3 & $1550(45.8)$ & $140(57.9)$ & $592(32.7)$ & $2917(64)$ \\
\hline 4 or 5 & $96(2.8)$ & $24(9.9)$ & $12(0.7)$ & $272(6.0)$ \\
\hline \multicolumn{5}{|l|}{ Wound classification } \\
\hline Clean & $3330(98.4)$ & $153(63.2)$ & $187(10.3)$ & 4340 (95.2) \\
\hline Clean/contaminated & $43(1.3)$ & $48(19.8)$ & $22(1.2)$ & $128(2.8)$ \\
\hline Contaminated & $5(0.1)$ & $37(15.3)$ & $0(0)$ & $20(0.4)$ \\
\hline Dirty/infected & $5(0.1)$ & $4(1.7)$ & $2(0.1)$ & $72(1.6)$ \\
\hline \multicolumn{5}{|l|}{ Origin of admission } \\
\hline Home & $2891(85.5)$ & $62(25.6)$ & $1767(97.6)$ & $2199(48.2)$ \\
\hline Emergency department & $395(11.7)$ & $5(2.1)$ & $25(1.4)$ & $1827(40.1)$ \\
\hline Chronic care facility & $1(0)$ & $1(0)$ & $0(0)$ & $14(0.3)$ \\
\hline Outside hospital $\dagger$ & $7(0.2)$ & $127(52.5)$ & $2(0.1)$ & $82(1.8)$ \\
\hline Other & $89(2.6)$ & $48(19.8)$ & $17(0.9)$ & $438(9.6)$ \\
\hline Inpatient & $3225(95.3)$ & $236(97.5)$ & $1767(97.6)$ & 4184 (91.8) \\
\hline \multicolumn{5}{|l|}{ Case status } \\
\hline Elective & $3105(91.8)$ & $101(41.7)$ & $1785(98.6)$ & 2432 (53.3) \\
\hline Emergency & $121(3.6)$ & $79(32.6)$ & $10(0.6)$ & $1241(27.2)$ \\
\hline Urgent & $157(4.6)$ & $62(25.6)$ & $16(0.9)$ & $887(19.5)$ \\
\hline
\end{tabular}

Data summarize a number of cases within a subgroup of each category (with percentages).

* Preterm was defined as any infant who was born at less than 37 weeks' gestation.

† Outside hospital includes NICU, PICU, and inpatient.

cardiac risk factors (Table 2). Nutritional support was predominantly found in defect repairs (17.4\%) and shunt and implant $(17.6 \%)$ categories. Pulmonary conditions varied across categories, with the craniotomy/craniectomy and laminectomy categories having the largest proportion of patients with asthma, the defect repair category having the greatest proportion of patients needing oxygen and ventilator support, and the shunts and implants category having the largest proportion of patients with chronic lung disease. The two most prevalent hematological and immunological conditions, cancer and preoperative steroid use, were the same for craniotomy/craniectomy, laminectomy, and shunt and implant categories, whereas for the defect repair category it was hematological disorder. Esophageal gastrointestinal disease was a common risk factor for laminectomy (16.7\%) and shunts and implants (19.4\%). More than $99.8 \%$ of patients did not have any renal conditions for all categories. Previous operation was among the top 
TABLE 2. Patient risk factors and measures of system efficiency and procedure complexity by NSQIP-Peds neurosurgical procedure categories

\begin{tabular}{|c|c|c|c|c|}
\hline Patient Risk Factors & $\begin{array}{c}\text { Craniotomy/ } \\
\text { Craniectomy (\%) }\end{array}$ & $\begin{array}{c}\text { Defect } \\
\text { Repairs (\%) }\end{array}$ & Laminectomy (\%) & $\begin{array}{c}\text { Shunts \& } \\
\text { Implants (\%) }\end{array}$ \\
\hline Total cases & $3383(100)$ & $242(100)$ & $1811(100)$ & $4560(100)$ \\
\hline \multicolumn{5}{|l|}{ Cardiovascular conditions } \\
\hline \multicolumn{5}{|l|}{ Congenital cardiac RF } \\
\hline No cardiac RF & $3226(95.4)$ & $208(86)$ & $1577(87.1)$ & $3932(86.2)$ \\
\hline Minor cardiac RF & $106(3.1)$ & $14(5.8)$ & $159(8.8)$ & $458(10)$ \\
\hline Major cardiac RF & $45(1.3)$ & $18(7.4)$ & $66(3.6)$ & $142(3.1)$ \\
\hline Severe cardiac RF & $6(0.2)$ & $2(0.8)$ & $9(0.5)$ & $28(0.6)$ \\
\hline Previous cardiac surgery & $56(1.7)$ & $2(0.8)$ & $73(4)$ & $255(5.6)$ \\
\hline \multicolumn{5}{|l|}{ Metabolic \& nutritional conditions } \\
\hline Diabetes & $10(0.3)$ & $0(0)$ & $3(0.2)$ & $13(0.3)$ \\
\hline Preop $10 \%$ weight loss & $50(1.5)$ & $0(0)$ & $37(2)$ & $141(3.1)$ \\
\hline Nutritional support & $88(2.6)$ & $42(17.4)$ & $79(4.4)$ & $804(17.6)$ \\
\hline \multicolumn{5}{|l|}{ Pulmonary conditions } \\
\hline Asthma & $184(5.4)$ & $0(0)$ & $115(6.4)$ & $301(6.6)$ \\
\hline Structural pulmonary abnormality & $122(3.6)$ & $5(2.1)$ & $87(4.8)$ & $276(6.1)$ \\
\hline Preop oxygen support & $75(2.2)$ & $29(12)$ & $26(1.4)$ & $375(8.2)$ \\
\hline Preop ventilator dependence & $71(2.1)$ & $23(9.5)$ & $13(0.7)$ & $262(5.7)$ \\
\hline Preop tracheostomy & $24(0.7)$ & $0(0)$ & $21(1.2)$ & $142(3.1)$ \\
\hline History of chronic lung disease & $36(1.1)$ & $2(0.8)$ & $40(2.2)$ & $520(11.4)$ \\
\hline Cystic fibrosis & $1(0)$ & $0(0)$ & $0(0)$ & $6(0.1)$ \\
\hline Preop pneumonia & $3(0.1)$ & $1(0.4)$ & $0(0)$ & $18(0.4)$ \\
\hline \multicolumn{5}{|c|}{ Hematological \& immunological conditions } \\
\hline Bleeding disorder & $19(0.6)$ & $0(0)$ & $8(0.4)$ & $38(0.8)$ \\
\hline Hematological disorder & $55(1.6)$ & $2(0.8)$ & $21(1.2)$ & $198(4.3)$ \\
\hline Preop transfusion & $33(1)$ & $1(0.4)$ & $1(0.1)$ & $58(1.3)$ \\
\hline Immune disorder & $37(1.1)$ & $0(0)$ & $9(0.5)$ & $40(0.9)$ \\
\hline Preop steroid use & $484(14.3)$ & $1(0.4)$ & $36(2)$ & $295(6.5)$ \\
\hline Current cancer & $791(23.4)$ & $0(0)$ & $46(2.5)$ & $248(5.4)$ \\
\hline Chemotherapy & $54(1.6)$ & $0(0)$ & $5(0.3)$ & $82(1.8)$ \\
\hline Radiotherapy & $12(0.4)$ & $0(0)$ & $0(0)$ & $25(0.5)$ \\
\hline Bone marrow transplant & $20(0.6)$ & $0(0)$ & $0(0)$ & $1(0)$ \\
\hline Organ transplant & $3(0.1)$ & $0(0)$ & $0(0)$ & $2(0)$ \\
\hline \multicolumn{5}{|l|}{ Neurological conditions } \\
\hline CNS structural abnormality & $1850(54.7)$ & $222(91.7)$ & $1427(78.8)$ & $3861(84.7)$ \\
\hline Cerebral palsy & $24(0.7)$ & $2(0.8)$ & $48(2.7)$ & $781(17.1)$ \\
\hline CNS tumor & $1116(33)$ & $0(0)$ & $88(4.9)$ & $373(8.2)$ \\
\hline Preop coma & $3(0.1)$ & $0(0)$ & $0(0)$ & $2(0)$ \\
\hline Neuromuscular disease & $165(4.9)$ & $24(9.9)$ & $205(11.3)$ & $570(12.5)$ \\
\hline Developmental delay & $515(15.2)$ & $4(1.7)$ & $399(22)$ & $1789(39.2)$ \\
\hline Preop seizure history & $381(11.3)$ & $3(1.2)$ & $78(4.3)$ & $1133(24.8)$ \\
\hline IVH Grade 3 or 4 & $38(1.1)$ & $6(2.5)$ & $13(0.7)$ & $979(21.5)$ \\
\hline Preop CVA & $90(2.7)$ & $1(0.4)$ & $27(1.5)$ & $517(11.3)$ \\
\hline \multicolumn{5}{|l|}{ Gastrointestinal conditions } \\
\hline ESOVAR & $282(8.3)$ & $9(3.7)$ & $302(16.7)$ & $883(19.4)$ \\
\hline Hepatobiliary pancreatic disease & $9(0.3)$ & $0(0)$ & $11(0.6)$ & $43(0.9)$ \\
\hline \multicolumn{5}{|l|}{ Renal conditions } \\
\hline Preop renal failure & $0(0)$ & $0(0)$ & $1(0.1)$ & $2(0)$ \\
\hline Preop dialysis & $1(0)$ & $0(0)$ & $0(0)$ & $3(0.1)$ \\
\hline
\end{tabular}


» CONTINUED FROM PAGE 365

TABLE 2. Patient risk factors and measures of system efficiency and procedure complexity by NSQIP-Peds neurosurgical procedure categories

\begin{tabular}{lcccc}
\hline \multicolumn{1}{c}{ Patient Risk Factors } & $\begin{array}{c}\text { Craniotomy/ } \\
\text { Craniectomy (\%) }\end{array}$ & $\begin{array}{c}\text { Defect } \\
\text { Repairs (\%) }\end{array}$ & Laminectomy (\%) & $\begin{array}{c}\text { Shunts \& } \\
\text { Implants (\%) }\end{array}$ \\
\hline Acuity of condition & & & & \\
\hline Preop open wound & $5(0.1)$ & $134(55.4)$ & $9(0.5)$ & $80(1.8)$ \\
\hline Previous op 30 days & $89(2.6)$ & $2(0.8)$ & $8(0.4)$ & $320(7)$ \\
\hline Preop sepsis 48 hrs & $14(0.4)$ & $1(0.4)$ & $1(0.1)$ & $132(2.9)$ \\
\hline Periop inotropic support & $34(1)$ & $2(0.8)$ & $9(0.5)$ & $39(0.9)$ \\
\hline Preop CPR & $7(0.2)$ & $1(0.4)$ & $0(0)$ & $14(0.3)$ \\
\hline Do not resuscitate & $1(0)$ & $0(0)$ & $0(0)$ & $9(0.2)$ \\
\hline
\end{tabular}

$\mathrm{CPR}=$ cardiopulmonary resuscitation; $\mathrm{CVA}$ = cerebrovascular accident; ESOVAR = esophageal gastric intestinal disease; IVH = intraventricular hemorrhage; RF = risk factor.

two most common acuity conditions for craniotomy/craniectomy, laminectomy, and shunts and implants, whereas for defect repair it was preoperative open wound (55.4\%).

The most common patient risk factor, CNS structural abnormality, was similar for all 4 procedure categories, with more than half of the patients having this comorbidity (Table 2). For all categories, at least 2 of the most common patient risk factors were neurological. Following a CNS structural abnormality, developmental delay was a common risk factor for all categories, except defect repair.

\section{System Efficiency and Procedural Complexity Measures}

For analysis of measures of system efficiency, we found that a longer duration from admission to operation was associated with any AE for craniotomy/craniectomy (mean duration 0.6 days for no $\mathrm{AE}$ vs 1.1 days for any $\mathrm{AE}, \mathrm{p}<$

TABLE 3. Efficiency and procedure complexity by NSQIP-Peds neurosurgical procedure categories

\begin{tabular}{|c|c|c|c|c|}
\hline \multirow[b]{2}{*}{ Clinical Factors $†$} & \multicolumn{2}{|c|}{$\mathrm{AE}$} & \multicolumn{2}{|c|}{ p Value* } \\
\hline & None & Any & Unadjusted & Adjusted \\
\hline \multicolumn{5}{|l|}{ Craniotomy/craniectomy } \\
\hline Admission to op (days) & $0.6 \pm 2.3$ & $1.1 \pm 4.3$ & $<0.001$ & 0.049 \\
\hline Length of hospital stay (days) & $4.5 \pm 4.7$ & $8.3 \pm 11.3$ & - & - \\
\hline wRVU & $32.2 \pm 5.3$ & $31.8 \pm 6.8$ & 0.046 & 0.503 \\
\hline Op time (mins) & $185.8 \pm 105.7$ & $246.4 \pm 146.3$ & $<0.001$ & $<0.001$ \\
\hline \multicolumn{5}{|l|}{ Defect repair } \\
\hline Admission to op (days) & $1.1 \pm 1.7$ & $3.2 \pm 17.7$ & 0.207 & NS \\
\hline Length of hospital stay (days) & $11.8 \pm 8.2$ & $18.5 \pm 14.3$ & - & - \\
\hline wRVU & $23.8 \pm 1.5$ & $24.0 \pm 1.5$ & 0.319 & NS \\
\hline Op time (mins) & $108.9 \pm 67.4$ & $115.1 \pm 70.0$ & 0.489 & NS \\
\hline \multicolumn{5}{|l|}{ Laminectomy } \\
\hline Admission to op (days) & $0.1 \pm 1.9$ & $0.8 \pm 5.3$ & 0.007 & 0.605 \\
\hline Length of hospital stay (days) & $2.9 \pm 3.1$ & $6.3 \pm 7.1$ & - & - \\
\hline wRVU & $22.7 \pm 4.0$ & $24.1 \pm 5.8$ & $<0.001$ & 0.174 \\
\hline Op time (mins) & $117.8 \pm 89.8$ & $188.3 \pm 129.1$ & $<0.001$ & $<0.001$ \\
\hline \multicolumn{5}{|l|}{ Shunts and implants } \\
\hline Admission to op (days) & $2.5 \pm 13.3$ & $9.1 \pm 23.2$ & $<0.001$ & 0.001 \\
\hline Length of hospital stay (days) & $5.3 \pm 11.2$ & $16.8 \pm 25.1$ & - & - \\
\hline wRVU & $10.7 \pm 3.5$ & $10.4 \pm 3.7$ & 0.049 & 0.001 \\
\hline Op time (mins) & $58.0 \pm 39.4$ & $61.2 \pm 56.5$ & 0.032 & 0.001 \\
\hline \multicolumn{5}{|c|}{$\begin{array}{l}\text { NS = variable did not meet the } p<0.05 \text { criterion for entry into the multivariable model; hence, no adjusted OR was computed. } \\
\text { Continuous variables expressed as mean } \pm S D \text {. Boldface type indicates statistical significance. } \\
\text { * Unadjusted } p \text { value generated from Student t-test analysis to identify significant differences in variable means between groups with no AE } \\
\text { versus groups with any AE. Adjusted } p \text { value generated by backward stepwise multivariable logistic regression analysis for any AE with variable } \\
\text { entry level } p<0.05 \text {. This adjustment accounts for other covariate risk factors that may affect the relationship between AEs and the variable of } \\
\text { interest. } \\
\dagger \text { Length of hospital stay is represented as a descriptive statistic, because it is partially an outcome measure. }\end{array}$} \\
\hline
\end{tabular}


0.001) and shunts and implants (mean duration 2.5 days for no AE vs 9.1 days for any AE, $p<0.001$ ) (Table 3). The mean length of hospital stay for craniotomy/craniectomy was 4.5 days for no AE versus 8.3 days for any AE. For defect repair, the mean duration was 11.8 days for no $\mathrm{AE}$ versus 18.5 days for any $\mathrm{AE}$, for laminectomy it was 2.9 days for no $\mathrm{AE}$ versus 6.3 days for any $\mathrm{AE}$, and for shunts and implants it was 5.3 days for no AE versus 16.8 days for any AE (Table 3). For procedural complexity, a longer total operation time was associated with any AE based on multivariable analysis for craniotomy/craniectomy (mean 185.8 minutes for no AE vs 246.4 minutes for any AE, $\mathrm{p}<0.001$ ), laminectomy (mean 117.8 minutes for no AE vs 188.3 minutes for any AE, $p<0.001$ ), and shunts and implants (mean 58.0 minutes for no AE vs 61.2 minutes for any AE, $\mathrm{p}=0.032$; Table 3). Work RVU was associated with any $\mathrm{AE}$ on multivariable analysis for the shunts and implants category (mean 10.7 for no AE vs 10.4 for any AE, $\mathrm{p}=0.049$; Table 3).

\section{Adverse Events}

Defect repair category had the highest occurrence of
AEs, with 52.9\% suffering at least 1 AE (Table 4). Laminectomy category had the lowest occurrence of AEs, with $11.1 \%$ of patients experiencing any AE. Mortality rates were under $1 \%$ in all categories, with the exception of defect repair, which had a mortality rate of $1.2 \%$. For all procedure categories except laminectomy, unplanned reoperation represented a significant portion of AEs. The highest rate of unplanned reoperation was seen in defect repairs (29.8\%) followed by shunts and implants (13.2\%) and craniotomy/craniectomy $(6.2 \%)$. The most common $\mathrm{AE}$ for craniotomy/craniectomy was bleeding requiring transfusion (24.2\%), which was relatively less common for the other procedure categories. In both defect repair and shunts and implants, ventilator dependence greater than 24 hours represented the most common AE at $24.4 \%$ and $5.8 \%$, respectively. For laminectomy, SSI and wound dehiscence were the most common AEs.

\section{Predictors of AEs}

Using findings from univariate analysis, we identified risk factors that were associated with any AE by multivariable analysis (Table 5). For craniotomy, 10 risk fac-

TABLE 4. Adverse events by NSQIP-Peds neurosurgical procedure categories

\begin{tabular}{|c|c|c|c|c|}
\hline Clinical Outcomes & Craniotomy/Craniectomy (\%) & Defect Repairs (\%) & Laminectomy (\%) & Shunts \& Implants (\%) \\
\hline Total cases & 3383 & 242 & 1811 & 4560 \\
\hline Any adverse event & $1091(32.2)$ & $128(52.9)$ & $201(11.1)$ & 889 (19.5) \\
\hline Mortality & $13(0.4)$ & $3(1.2)$ & $1(0.1)$ & $18(0.4)$ \\
\hline Unplanned reop & $209(6.2)$ & $72(29.8)$ & $73(4)$ & $602(13.2)$ \\
\hline SSI & $62(1.8)$ & $12(5)$ & $67(3.7)$ & $114(2.5)$ \\
\hline Superficial & $28(0.8)$ & $6(2.5)$ & $47(2.6)$ & $21(0.5)$ \\
\hline Deep & $5(0.1)$ & $2(0.8)$ & $14(0.8)$ & $7(0.2)$ \\
\hline Organ-space infection & $29(0.9)$ & $4(1.7)$ & $6(0.3)$ & $89(2)$ \\
\hline Composite morbidity & $1022(30.2)$ & $94(38.8)$ & $185(10.2)$ & $489(10.7)$ \\
\hline Superficial SSI & $28(0.8)$ & $6(2.5)$ & $47(2.6)$ & $21(0.5)$ \\
\hline Deep SSI & $5(0.1)$ & $2(0.8)$ & $14(0.8)$ & $7(0.2)$ \\
\hline Organ-space infection & $29(0.9)$ & $4(1.7)$ & $6(0.3)$ & $89(2)$ \\
\hline Superficial wound dehiscence & $33(1)$ & $13(5.4)$ & $37(2)$ & $26(0.6)$ \\
\hline Deep wound dehiscence & $33(1)$ & $18(7.4)$ & $43(2.4)$ & $25(0.5)$ \\
\hline SIRS/sepsis/septic shock & $25(0.7)$ & $1(0.4)$ & $10(0.6)$ & $37(0.8)$ \\
\hline Unplanned reintubation & $32(0.9)$ & $10(4.1)$ & $4(0.2)$ & $37(0.8)$ \\
\hline Pneumonia & $10(0.3)$ & $0(0)$ & $1(0.1)$ & $21(0.5)$ \\
\hline Ventilator dependence $>24 \mathrm{hrs}$ & $177(5.2)$ & $59(24.4)$ & $24(1.3)$ & $265(5.8)$ \\
\hline Pulmonary embolism & $0(0)$ & $0(0)$ & $0(0)$ & $0(0)$ \\
\hline Cardiac arrest & $1(0)$ & $0(0)$ & $0(0)$ & $5(0.1)$ \\
\hline Urinary tract infection & $22(0.7)$ & $5(2.1)$ & $34(1.9)$ & $33(0.7)$ \\
\hline Progressive renal insufficiency & $0(0)$ & $0(0)$ & $0(0)$ & $2(0)$ \\
\hline Acute renal failure & $0(0)$ & $0(0)$ & $0(0)$ & $0(0)$ \\
\hline Cerebrovascular accident & $25(0.7)$ & $0(0)$ & $0(0)$ & $13(0.3)$ \\
\hline Coma for $>24 \mathrm{hrs}$ & $2(0.1)$ & $0(0)$ & $0(0)$ & $1(0)$ \\
\hline Seizure & $33(1)$ & $3(1.2)$ & $1(0.1)$ & $33(0.7)$ \\
\hline Nerve injury & $7(0.2)$ & $0(0)$ & $1(0.1)$ & $1(0)$ \\
\hline Venous thromboembolism & $6(0.2)$ & $0(0)$ & $0(0)$ & $7(0.2)$ \\
\hline Intra/postop blood transfusion & $820(24.2)$ & $10(4.1)$ & $21(1.2)$ & $53(1.2)$ \\
\hline
\end{tabular}

SIRS = systemic inflammatory response syndrome. 
TABLE 5. Multivariable analysis of predictors of any adverse event by NSQIP-Peds neurosurgical procedure categories

\begin{tabular}{|c|c|c|c|c|c|c|c|c|c|c|c|c|}
\hline \multirow[b]{2}{*}{ Risk Factor } & \multicolumn{3}{|c|}{ Craniotomy/Craniectomy } & \multicolumn{3}{|c|}{ Defect Repair } & \multicolumn{3}{|c|}{ Laminectomy } & \multicolumn{3}{|c|}{ Shunts/Implants } \\
\hline & AOR & $95 \% \mathrm{Cl}$ & p Value & AOR & $95 \% \mathrm{Cl}$ & $\mathrm{p}$ Value & AOR & $95 \% \mathrm{Cl}$ & $\mathrm{p}$ Value & AOR & $95 \% \mathrm{Cl}$ & $p$ Value \\
\hline Male & & & & & & & 0.7 & $0.5-0.9$ & 0.012 & & & \\
\hline \multicolumn{13}{|l|}{ Age } \\
\hline$<29$ days & 9.0 & $1.9-42.3$ & 0.005 & & & & & & & & & \\
\hline 29-364 days & 26.3 & $19.1-36.1$ & $<0.001$ & & & & & & & & & \\
\hline $1-5 \mathrm{yrs}$ & 5.0 & $3.7-6.8$ & $<0.001$ & & & & & & & & & \\
\hline $6-12$ yrs & 1.5 & $1.1-2.1$ & 0.014 & & & & & & & & & \\
\hline $13-18$ yrs & 1.0 & & Ref & & & & & & & & & \\
\hline ASA Class 4 or 5 & 2.3 & $1.3-4.1$ & 0.004 & 3.8 & $1.3-11.0$ & 0.014 & & & & & & \\
\hline \multicolumn{13}{|l|}{ Admission origin } \\
\hline Home & & & & 1.0 & & Ref & 1.0 & & Ref & 1.0 & & Ref \\
\hline Emergency department & & & & 0.4 & $0.0-3.9$ & 0.408 & 2.5 & $1.0-6.4$ & 0.049 & 1.3 & $1.1-1.6$ & 0.01 \\
\hline Chronic care & & & & 2.2 & $1.1-4.3$ & 0.018 & 11.7 & $4.1-33.2$ & $<0.001$ & 1.9 & $0.6-6.2$ & 0.271 \\
\hline Hospital $^{*}$ & & & & 2.3 & $1.0-5.1$ & 0.048 & 14.3 & $0.9-230.9$ & 0.062 & 1.8 & $1.4-2.4$ & $<0.001$ \\
\hline Inpatient & & & & & & & & & & 1.6 & $1.2-2.2$ & 0.005 \\
\hline \multicolumn{13}{|l|}{ Case status } \\
\hline Elective & 1.0 & & Ref & & & & & & & 1.0 & & Ref \\
\hline Emergency & 1.7 & $1.1-2.7$ & 0.027 & & & & & & & 1.3 & $1.1-1.6$ & 0.005 \\
\hline Urgent & 1.8 & $1.2-2.6$ & 0.0006 & & & & & & & 1.1 & $0.9-1.3$ & 0.476 \\
\hline \multicolumn{13}{|l|}{ Congenital CRF } \\
\hline None & & & & & & & & & & 1.0 & & Ref \\
\hline Minor & & & & & & & & & & 1.3 & $1.0-1.6$ & 0.041 \\
\hline Major & & & & & & & & & & 1.4 & $0.9-2.1$ & 0.115 \\
\hline Severe & & & & & & & & & & 1.5 & $0.6-3.6$ & 0.385 \\
\hline Preop $10 \%$ weight loss & 2.2 & $1.1-4.5$ & 0.035 & & & & & & & & & \\
\hline Nutritional support & & & & & & & & & & 2.0 & $1.6-2.4$ & $<0.001$ \\
\hline Preop oxygen support & & & & & & & & & & 1.5 & $1.1-2.0$ & 0.005 \\
\hline Preop ventilator dependence & 6.9 & $3.5-13.5$ & $<0.001$ & 4.3 & $1.3-13.8$ & 0.016 & 69.8 & $8.7-562.6$ & $<0.001$ & 6.4 & $4.5-9.0$ & $<0.001$ \\
\hline Preop tracheostomy & 4.9 & $1.4-17.3$ & 0.015 & & & & & & & & & \\
\hline Preop transfusion & & & & & & & & & & 3.4 & $1.7-6.9$ & 0.001 \\
\hline Chemotherapy & 3.0 & $1.5-5.7$ & 0.001 & & & & & & & & & \\
\hline CNS structural abnormality & & & & 0.3 & $0.1-0.9$ & 0.032 & & & & & & \\
\hline Cerebral palsy & & & & & & & & & & 0.8 & $0.6-0.9$ & 0.009 \\
\hline CNS tumor & & & & & & & & & & 1.5 & $1.1-1.9$ & 0.003 \\
\hline Development delay & 1.5 & $1.2-2.0$ & 0.001 & & & & 1.9 & $1.3-2.7$ & & & & \\
\hline Preop seizure history & 0.5 & $1.2-2.0$ & $<0.001$ & & & & & & & & & \\
\hline IVH Grade 3 or 4 & 2.8 & $1.3-6.2$ & 0.01 & & & & & & & & & \\
\hline Preop sepsis $48 \mathrm{hr}$ & & & & & & & & & & 1.9 & $1.3-2.7$ & 0.02 \\
\hline Do not resuscitate & & & & & & & & & & 5.0 & $1.1-22.3$ & 0.037 \\
\hline Admit to op (days) & $1.04 \dagger$ & & 0.049 & & & & & & & $1.01 \dagger$ & & 0.001 \\
\hline wRVU & & & & & & & & & & $0.96 \dagger$ & & 0.001 \\
\hline Total op time (min) & $1.01 \dagger$ & & $<0.001$ & & & & $1.01 \dagger$ & & $<0.001$ & $1.01 \dagger$ & & 0.001 \\
\hline
\end{tabular}

CRF = cardiac risk factor; Ref $=$ reference.

Adjusted $\mathrm{OR}, 95 \% \mathrm{Cl}$, and $\mathrm{p}$ value generated from multivariable logistic regression of 30-day postoperative any AEs with stepwise elimination of variables not significant at the $p<0.05$ level. Multivariable models were controlled for demographic, patient risk factor, and continuous variable covariates listed in Tables 1 and 2 . Empty cells represent risk factors that were not significant at the $p<0.05$ level on stepwise multivariable analysis.

* Outside hospital.

$\dagger$ System efficiency and procedure complexity measures are continuous variables. This explains the nature of the marginal increase in odds of incurring any AE for each variable unit increase (i.e., days, wRVU, and minutes). 
tors (in decreasing order of adjusted OR [AOR]) were associated with any AE: age (29-364 days, AOR 26.3; < 29 days, AOR 9.0; $1-5$ years, AOR 5.0; 6-12 years, AOR 1.5; reference 13-18 years), ventilator dependence (AOR 6.9), preoperative tracheostomy (AOR 4.9), chemotherapy (AOR 3.0), intraventricular hemorrhage Grade 3 or 4 (AOR 2.8), ASA Class 4 or 5 (AOR 2.3; reference ASA Class 1-3), preoperative weight loss (AOR 2.2), case status (urgent, AOR 1.8; emergency, AOR 1.7; reference, elective), developmental delay (AOR 1.5), and seizure history (AOR 0.5). Four patient risk factors (in decreasing order of AOR) were associated with any AE for defect repair: ventilator dependence (AOR 4.3), ASA Class 4 or 5 (AOR, 3.8; reference ASA Class 1-3), origin of admission (outside hospital, AOR 2.3; chronic care facility, AOR 2.2; reference, home), and CNS structural abnormality (AOR 0.3). Four patient risk factors (in decreasing order of AOR) were associated with any AE for laminectomy: ventilator dependence (AOR 69.8), origin of admission (outside hospital, AOR 14.3; chronic care facility, AOR 11.7; emergency department, AOR 2.5; reference, home), developmental delay (AOR 1.9), and female sex (AOR 1.3). Eleven patient risk factors (in decreasing order of AOR) were associated with any $\mathrm{AE}$ for shunts and implants: ventilator dependence (AOR 6.4), do not resuscitate status (AOR 5.0), preoperative transfusion (AOR 3.4), nutritional support (AOR 2.0), sepsis (AOR 1.9), origin of admission (chronic care facility, AOR 1.9; outside hospital, AOR 1.8; emergency department, AOR 1.3; reference, home), CNS tumor (AOR 1.5), oxygen supplementation (AOR 1.5), cardiac risk factors (severe, AOR 1.5; major, AOR 1.4; minor, AOR 1.3; reference, no cardiac risk factors), inpatient (AOR 1.6), and case status (emergency, AOR 1.3; urgent, AOR 1.1; reference, elective). With regards to system efficiency, after accounting for all covariates, admission to operation time was retained as a significant predictor of AEs for the following procedure categories: craniotomy/ craniectomy and shunts/implants. Use of this adjustment demonstrates that independent of the patient's risk factor level, a longer admission to operation time significantly correlated with any AE. For procedure complexity, operation time significantly correlated with any AE for craniotomy/craniectomy, laminectomy, and shunts/implants procedure category, independent of the patient's risk factor (Table 3).

\section{Laboratory Data}

For all procedure categories, except laminectomy, only select laboratory variables (hematocrit, platelets, and WBC) had fewer than 33\% missing data and were included in our analysis. Hematocrit (mean $\pm \mathrm{SD}$ gm/dl) was associated with any AE by univariate analysis for craniotomy/craniectomy (37.6 \pm 4.1 for no $\mathrm{AE}$ vs $35.3 \pm 4.1$ for any AE, p < 0.001) and shunts and implants $(37.7 \pm 5.3$ for no $\mathrm{AE}$ vs $36.4 \pm 6.3$ for any AE, $\mathrm{p}<0.001$ ). Platelet (mean $\pm \mathrm{SD} \times 10^{3} / \mu \mathrm{l}$, was associated with any $\mathrm{AE}$ on univariate analysis for craniectomy $(301.3 \pm 96.4$ for no AE vs 345.8 \pm 113.6 for any AE, p < 0.001) and defect repair $(270.9$ \pm 110 for no $\mathrm{AE}$ vs $232.6 \pm 72.8$ for any AE, $\mathrm{p}=0.004$ ). WBC count (mean $\pm \mathrm{SD} \times 10^{3} / \mu \mathrm{l}$ ) was associated with any AE on univariate analysis for craniotomy/craniectomy
$(8.0 \pm 3.2$ for no AE vs $9.7 \pm 4.0$ for any AE, $\mathrm{p}<0.001)$ and shunts and implants $(9.4 \pm 4.0$ for no AE vs $10.5 \pm 5.1$ for any $\mathrm{AE}, \mathrm{p}<0.001)$.

\section{Discussion}

To our knowledge, studies using the ACS NSQIP-Peds platform to describe perioperative mortality and morbidity for various pediatric neurosurgical procedure categories have been limited. We presented a broad national landscape of patients who underwent an NSQIP-Peds targeted pediatric neurosurgical procedure between 2012 and 2014. Our analysis should help neurosurgeons, intensivists, and hospital administrative staff guide clinical care and QI initiatives.

We found that the rate of AEs varied widely by procedure category, with the highest rates following defect repair followed by craniotomy/craniectomy, shunts and implants, and laminectomy. These rates are higher than previously reported by Saito et al. in their overview of all NSQIPPeds procedures, ${ }^{15}$ although their analyses did not include the occurrences of unplanned reoperation. There is an increased emphasis to include unplanned reoperation measures into surgical quality assessments, which is especially pertinent in pediatric and adult neurosurgery where high rates of reoperation often are required to address important complications, such as postoperative intracranial bleeding and shunt failures.,10,19 In addition, we found the composite morbidity for craniotomy/craniectomy and defect repairs higher than previously reported..$^{15}$ These 2 procedures had high rates of bleeding, requiring transfusion and ventilator dependence $>24$ hours. Although the overall rate of AEs following laminectomy was relatively low, the major contributors to composite morbidity were SSI and wound dehiscence, which represented a greater proportion than in other procedure categories. For the shunts and implants, the major contributor to its postoperative complications was unplanned reoperation.

We found the frequency of unplanned reoperations highest in the defect repair (29.8\%) and shunt and implant $(13.2 \%)$ categories, suggesting that these procedures may carry significant risks of neurosurgical complications. Our results are consistent with a similar high rate of unplanned reoperation following other types of adult and pediatric neurosurgical procedures. ${ }^{8}$ In a study looking at risk factors for unplanned readmission in pediatric neurosurgery, Sherrod et al. found shunts and implants (16.8\%) and myelomeningocele repair (14.4\%) to have the 2 highest rates. ${ }^{17}$ Although only looking at unplanned readmission may fail to capture patients having AEs needing reoperation during their initial stay, this finding supports our conclusion that these 2 procedure categories are associated with a significantly higher risk of subsequent unplanned interventions. In their study, SSIs were found to be the most significant risk factor for unplanned readmission. Interpreting our results in this context allows the identification of the specific risk factors for SSIs and other AEs across varying procedure categories, which provides health care teams to better anticipate and manage the risk of these AEs and unplanned readmission and reoperation.

A comparison of mortality and morbidity with that in 
other pediatric surgical subspecialties would be helpful to guide multidisciplinary QI initiatives and quality benchmarking. However, to date the most comprehensive assessment using NSQIP-Peds has been limited to the specialty level of analysis, and more granular assessment (by procedures) or procedure class is needed to guide QI efforts. Saito et al. found that pediatric neurosurgery had the highest rate of morbidity, not accounting for unplanned reoperation, across all pediatric subspecialties: general surgery (7.0), neurosurgery (13.6\%), orthopedic surgery (2.4\%), otolaryngology $(2.0 \%)$, plastic surgery $(10.0 \%)$, and urological surgery $(3.1 \%))^{15}$

There are several notable differences in the AE types between pediatric and adult neurosurgical patients. For adult neurosurgical patients, common AEs include postoperative pneumonia, ventilator dependence $>48$ hours, urinary tract infections, deep venous thrombosis, and reoperation within 30 days..$^{14}$ In contrast, children have a different profile of complication types as well as risk factors. Mortality is generally a rare occurrence in the pediatric neurosurgical setting, similar to other pediatric surgical subspecialties. ${ }^{2,3,13}$ Our mortality findings are consistent with previously reported figures of $0.8 \%$ for pediatric neurosurgery. ${ }^{15}$ The higher rate $(1.2 \%)$ noted in defect repair, as compared with below $1.0 \%$ noted in the other 3 procedure categories, is likely due to the low occurrence $(n=3)$ and low case volume $(\mathrm{n}=242)$.

Efficiency measures such as delays in care are increasingly recognized as important attributes of health care quality. We found that patients who suffered AEs following craniotomy/craniectomy as well as shunt and implant procedures were more likely to have had a delay in surgery. Although this association does not confirm causation, this is not a trivial finding, because the presence of any $\mathrm{AE}$ is also associated with a longer length of hospitalization. Further analysis may help identify whether these differences result from the patient's risk profile, clinical management, or operational efficiencies of a hospital system. Some of these outcomes may be related to procedure complexity, as we noted a longer total operation time in patients who had an AE compared with those who did not experience any $\mathrm{AE}$ for most categories. An association between AEs and wRVU was only observed in shunts and implants, suggesting that wRVU may not serve as an accurate predictor of postoperative complication for most procedures. Measures such as wRVU values themselves have too many confounders to use for prediction of complications, because wRVUs are not only based on a physician's time and effort, but also incorporate input from procedure expense and professional liability insurance. In most studies, the use of wRVU alone for surgical risk prediction has not shown consistency, because it often does not account for anatomical or procedural variations among operations with similar RVUs. ${ }^{1}$ Similar to most studies, our study demonstrated that despite the use of wRVU within a distinct CPT procedure range, the case mix complexity was not accurately captured in risk prediction. . $^{3,11}$

Many of the predictors of AEs were expected, such as ASA Class 4 or 5 and preoperative ventilator dependence, because they are considered high-risk comorbidities as noted in other surgical specialties. ${ }^{15}$ However, many neu- rological risk factors (such as neuromuscular disease) that were associated with AEs by univariate analysis did not retain their significance on multivariable analysis. Future modeling that accounts for procedure-specific risk factors, efficiency measures, and procedural complexities may serve to be a more holistic risk prediction model that has better clinical value. Many of the predictors of AEs are reflective of underlying patient characteristics and are not readily remediable, although they may serve to delineate the risk profile within a specific patient population. In addition, having a full understanding of the patient's risk profile preoperatively may allow medical teams to better anticipate and prepare for specific postoperative AEs.

Several limitations exist in this study, many of which are inherent to analysis of a large surgical outcome data set. A major strength of NSQIP-Peds is its protocolized sampling and use of standardized outcome definitions. However, several limitations exist to these processes as they pertain to pediatric neurosurgical patients. First, epilepsy CPT codes were only recently added to NSQIP-Peds collection protocols, and future studies should investigate the outcomes in this increasingly common category. Second, many important AEs experienced by neurosurgical patients are not directly captured within NSQIP-Peds, such as shunt infections, meningitis, CSF leakage, intracranial bleeding, and seizures. Similarly, certain AEs, such as postoperative ventilator dependence $>24$ hours, may be normal clinical practice for some children undergoing neurosurgical procedures, and are therefore not an appropriate measure of quality. For example, some neonates have brain swelling or brainstem abnormality as part of their defect repair and are commonly kept intubated longer than 24 hours. Third, many of the perioperative complications often observed in neurosurgical patients, such as neurological impairment, may be either permanent or transient, resulting in dramatically different long-term outcomes. Because NSQIP-Peds only collects outcomes to 30 days following surgery, there are no means to delineate whether a neurological deficit is permanent or resolves over time. Fourth, although unplanned reoperations are increasingly being incorporated into measures of hospital and surgical quality, concerns have been raised in regards to the usefulness of this outcome in the NSQIP program. Unplanned reoperation (as defined by NSQIP) is the absence of any preoperative or intraoperative documentation that specifies the need for a patient to return to the operating room. Auditing of NSQIP vascular surgery cases has shown that more than $50 \%$ of operations coded as unplanned are related to errors in documentation, rather than complications such as bleeding or infection. ${ }^{8}$ Therefore, interpretation of our unplanned reoperation rate should be consistent with these constraints. Finally, because all AEs were aggregated together as the composite outcome measure for our multivariable models, this approach does not delineate associations between risk factors and complications of varying severity. Future studies could explore these associations through varying complication severity weights for each AE.

\section{Conclusions}

NSQIP-Peds offers a broad national overview of cur- 
rent expected perioperative outcomes following neurosurgical procedures in children. Although we found that the overall rates of mortality following pediatric neurosurgical procedures are low, there is a relatively high rate of AEs for some procedure categories, offering several areas for targeted QI interventions, such as the use of care bundles to decrease the rate of SSIs as has been described in several surgical specialties. In particular, significant delays to operation are associated with increased rates of AEs, suggesting a potential role for system level improvements. Although there are several limitations to our current use of NSQIP-Peds, the use of this data set has transformed our understanding of the outcomes and quality of neurosurgical care for children.

\section{Acknowledgments}

This study was made possible by the Duke University Graduate School Scholarship provided to Benjamin J. Kuo.

\section{References}

1. Bergersen L, Gauvreau K, McElhinney D, Fenwick S, Kirshner D, Harding J, et al: Capture of complexity of specialty care in pediatric cardiology by work RVU measures. Pediatrics 131:258-267, 2013

2. Bruny JL, Hall BL, Barnhart DC, Billmire DF, Dias MS, Dillon PW, et al: American College of surgeons national surgical quality improvement program pediatric: a beta phase report. J Pediatr Surg 48:74-80, 2013

3. Dillon P, Hammermeister K, Morrato E, Kempe A, Oldham $\mathrm{K}$, Moss L, et al: Developing a NSQIP module to measure outcomes in children's surgical care: opportunity and challenge. Semin Pediatr Surg 17:131-140, 2008

4. Hall BL, Hamilton BH, Richards K, Bilimoria KY, Cohen ME, Ko CY: Does surgical quality improve in the American College of Surgeons National Surgical Quality Improvement Program: an evaluation of all participating hospitals. Ann Surg 250:363-376, 2009

5. Keswani A, Uhler LM, Bozic KJ: What quality metrics is my hospital being evaluated on and what are the consequences? J Arthroplasty 31:1139-1143, 2016

6. Khuri SF, Daley J, Henderson W, Barbour G, Lowry P, Irvin G, et al: The National Veterans Administration Surgical Risk Study: risk adjustment for the comparative assessment of the quality of surgical care. J Am Coll Surg 180:519-531, 1995

7. Khuri SF, Henderson WG, Daley J, Jonasson O, Jones RS, Campbell DA Jr, et al: Successful implementation of the Department of Veterans Affairs' National Surgical Quality Improvement Program in the private sector: the Patient Safety in Surgery study. Ann Surg 248:329-336, 2008

8. Lin Y, Meguid RA, Henderson WG, Hammermeister K, Wild TT, Spruiell MD, et al: Unplanned return to the operating room: an institutional analysis to identify areas for improvement. J Am Coll Surg 221 (Suppl 1):S79-S80, 2015

9. McLaughlin N, Jin P, Martin NA: Assessing early unplanned reoperations in neurosurgery: opportunities for quality improvement. J Neurosurg 123:198-205, 2015

10. Mukerji N, Jenkins A, Nicholson C, Mitchell P: Unplanned reoperation rates in pediatric neurosurgery: a single center experience and proposed use as a quality indicator. J Neurosurg Pediatr 9:665-669, 2012

11. Raval MV, Cohen ME, Ingraham AM, Dimick JB, Osborne $\mathrm{NH}$, Hamilton BH, et al: Improving American College of Surgeons National Surgical Quality Improvement Program risk adjustment: incorporation of a novel procedure risk score. J Am Coll Surg 211:715-723, 2010

12. Raval MV, Dillon PW, Bruny JL, Ko CY, Hall BL, Moss RL, et al: American College of Surgeons National Surgical Quality Improvement Program Pediatric: a phase 1 report. J Am Coll Surg 212:1-11, 2011

13. Raval MV, Dillon PW, Bruny JL, Ko CY, Hall BL, Moss RL, et al: Pediatric American College of Surgeons National Surgical Quality Improvement Program: feasibility of a novel, prospective assessment of surgical outcomes. J Pediatr Surg 46:115-121, 2011

14. Rolston JD, Han SJ, Lau CY, Berger MS, Parsa AT: Frequency and predictors of complications in neurological surgery: national trends from 2006 to 2011. J Neurosurg 120:736745, 2014

15. Saito AJM, Chen E: Risk-adjusted hospital outcomes for children's surgery. Pediatrics 132:e677-e688, 2013

16. Shah RK, Stey AM, Jatana KR, Rangel SJ, Boss EF: Identification of opportunities for quality improvement and outcome measurement in pediatric otolaryngology. JAMA Otolaryngol Head Neck Surg 140:1019-1026, 2014

17. Sherrod BA, Johnston JM, Rocque BG: Risk factors for unplanned readmission within 30 days after pediatric neurosurgery: a nationwide analysis of 9799 procedures from the American College of Surgeons National Surgical Quality Improvement Program. J Neurosurg Pediatr 18:350-362, 2016

18. Shiloach M, Frencher SK Jr, Steeger JE, Rowell KS, Bartzokis K, Tomeh MG, et al: Toward robust information: data quality and inter-rater reliability in the American College of Surgeons National Surgical Quality Improvement Program. J Am Coll Surg 210:6-16, 2010

19. Zheng XR, Chen T, Yang YF, Rao W, Wang GY, Zhang SH, et al: Unplanned reoperations in neurosurgical patients due to postoperative bleeding: a single-center experience and literature review. Medicine (Baltimore) 94:e739, 2015

\section{Disclosures}

The authors report no conflict of interest concerning the materials or methods used in this study or the findings specified in this paper.

\section{Author Contribution}

Conception and design: Rice, Kuo, Haglund. Acquisition of data: Rice, Kuo. Analysis and interpretation of data: Rice, Kuo, Vissoci, Smith. Drafting the article: Kuo. Critically revising the article: Rice, Kuo, Egger, Smith, Grant, Haglund. Reviewed submitted version of manuscript: Rice, Kuo, Vissoci, Smith, Grant, Haglund. Approved the final version of the manuscript on behalf of all authors: Rice. Statistical analysis: Kuo, Egger. Study supervision: Rice.

\section{Correspondence}

Henry E. Rice, Division of Pediatric Surgery, Duke University Medical Center, Box 3815, Durham, NC 27710. email: henry. rice@dm.duke.edu. 